\title{
Trepadeiras do Parque Estadual da Serra da Tiririca, Rio DE JANEIRO, B RASIL ${ }^{1}$
}

\author{
Ana Angélica Monteiro de Barros², Leonor de Andrade Ribas ${ }^{3}$ \\ \& Dorothy Sue Dunn Araujo ${ }^{4}$
}

\begin{abstract}
Resumo
(Trepadeiras do Parque Estadual da Serra da Tiririca, Rio de Janeiro, Brasil) As trepadeiras têm alta representatividade em várias formações vegetais, correspondendo a cerca de $25 \%$ da diversidade taxonômica das florestas tropicais. Ainda assim, elas são mal representadas em levantamentos florísticos. Estudos na floresta ombrófila densa são raros e o presente trabalho vem contribuir para o conhecimento da flora de trepadeiras dessa formação vegetal. Foram feitas coletas no Parque Estadual da Serra da Tiririca, localizado nos municípios de Niterói e Maricá, estado do Rio de Janeiro. O levantamento florístico registrou 38 famílias, 107 gêneros e 215 espécies. Leguminosae (com 29 espécies), Sapindaceae (23), Bignoniaceae (22), Malpighiaceae (19) e Apocynaceae (15) foram as famílias com maior número de espécies, totalizando 50,5\% das espécies registradas. Houve um predomínio de trepadeiras lenhosas e de formas volúveis. O grande número de espécies encontradas é possivelmente resultado de um mosaico de vegetações em diferentes estádios sucessionais resultante do processo de uso e abandono da terra.
\end{abstract}

Palavras-chave: diversidade, florística, Mata Atlântica.

\section{Abstract}

(Climbers of the Serra da Tiririca State Park, Rio de Janeiro, Brazil) Climbers are highly represented in several vegetation types, corresponding to nearly $25 \%$ of tropical forest taxonomic diversity, but they are very poorly represented in floristic surveys. Studies of dense ombrophilous forests are rare and this work aims at contributing to the knowledge of climbers in this plant formation. The survey was undertaken in the Serra da Tiririca State Park, located between Niterói and Maricá municipalities, Rio de Janeiro state. The floristic survey listed 38 families, 107 genera, and 215 species. Leguminosae (with 29 species), Sapindaceae (23), Bignoniaceae (22), Malpighiaceae (19), and Apocynaceae (15) are the families better represented in number of species, accounting for $50.5 \%$ of the total number of species reported. Woody, twining climbers predominated in the inventory. The high number of species found is possibly related to a mosaic of vegetation in different successional stages resulted from the process of use and abandonment of land.

Key words: diversity, Atlantic rainforest, floristics.

\section{INTRODUÇÃo}

As trepadeiras apresentam alta representatividade em várias formações vegetacionais, correspondendo a cerca de $25 \%$ da diversidade taxonômica das florestas tropicais, sendo um importante componente florístico, estrutural e funcional (Gentry 1991; Engel et al. 1998). Possuem grande variedade de formas e tamanhos, uma vez que cerca da metade das famílias de angiospermas possuem representantes com hábito trepador (Peñalosa
1984). Normalmente, a abundância de plantas trepadeiras é relacionada às bordas de florestas, margens de cursos de água, clareiras e áreas sob influência antrópica, contudo também representam um componente significativo da vegetação do interior das matas.

Rezende \& Ranga (2005) enfatizam que, apesar da reconhecida importância ecológica, o enfoque dos estudos com trepadeiras tem sido pequeno. Gentry (1991) as coloca como a forma de vida menos coletada, o que pode ser comprovado

Artigo recebido em 10/2008. Aceito para publicação em 06/2009.

${ }^{1}$ Parte da tese de Doutoradodo primeiro autor. Programa de Pós-Graduação em Botânica da Escola Nacional de Botânica, Instituto de Pesquisas Jardim Botânico do Rio de Janeiro.

${ }^{2}$ Universidade do Estado do Rio de Janeiro, Faculdade de Formação de Professores, Depto. Ciências, R. Francisco Portela 794, 24.435-000, São Gonçalo, RJ, Brasil. anaangbarros@gmail.com

${ }^{3}$ IBAMA, SUPES/RJ. Praça XV 42, $8^{\circ}$ andar, 20010-010, Rio de Janeiro, RJ, Brasil.

${ }^{4}$ Universidade Federal do Rio de Janeiro, CCS, Instituto de Biologia, Depto. Ecologia, Lab. Ecologia Vegetal, C.P. 68020, 21941-590, Rio de Janeiro, RJ, Brasil. 
pela pouca representatividade de espécimes de trepadeiras nos herbários. Tal fato pode estar relacionado às dificuldades metodológicas, uma vez que as trepadeiras atingem grandes alturas no dossel, sendo difícil a visualização de seus ramos férteis. Quando o material coletado é estéril, chaves com características vegetativas (Vaz \& Vieira 1994; Gentry 1993; Ribeiro et al. 1999) podem auxiliar na sua identificação. Nesse sentido, estudos anatômicos, como os de Carlquist (1991), Araújo \& Costa (2006, 2007) e Brandes (2007), também podem representar bases importantes para a identificação de espécies de trepadeiras.

A partir da década de 1990, os estudos florísticos e fitossociológicos desenvolvidos no Brasil têm dado um enfoque maior às trepadeiras em diferentes formações vegetacionais: florestas estacionais semideciduais (Bernacci \& LeitãoFilho 1996; Stranghetti \& Ranga 1998; Morellato \& Leitão-Filho 1998; Rezende \& Ranga 2005; Tibiriçá et al. 2006), florestas úmidas (Prance 1994; Oliveira et al. 2008) e cerrado (Weiser 2001). No estado do Rio de Janeiro, destaca-se o trabalho pioneiro de Lima et al. (1997)na floresta ombrófila densa montana e altomontana da Reserva Biológica de Macaé de Cima e a chave de identificação para famílias de Vaz \& Vieira (1994).

Apesar de sua importância e diversidade nas florestas tropicais (Putz 1984; Peixoto \& Gentry 1990; Leitão-Filho 1995; Sá 1996; Lombardi et al. 1999; Tibiriçáet al. 2006), plantas com hábito trepador ainda são pouco enfocadas em trabalhos na Mata Atlântica (Vaz \& Vieira 1994; Lima et al. 1997; Tabanez \& Viana 2000; Sá 2006). Este estudo visa minimizar essa lacuna, caracterizando a composição florística das trepadeiras em formações remanescentes da floresta ombrófila densa submontana litorânea do Parque Estadual da Serra da Tiririca no estado do Rio de Janeiro.

\section{Material e Métodos}

\section{Área de estudo}

O Parque Estadual da Serra da Tiririca (PEST) está localizado entre os municípios de Niterói e Maricá, no estado do Rio de Janeiro

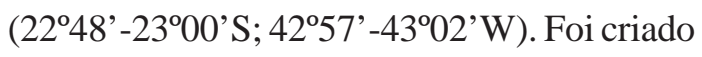
pela Lei Estadual n 1901/91 de 29 de novembro de 1991, tendo seus "limites em estudo" estabelecidos pelo Decreto $\mathrm{n}^{\circ} 18.598$ de 19 de maio de 1993 para uma área de 2.400 ha (Pontes 1987). A Lei Estadual $n^{\circ} 5079$, de 03 de setembro de 2007, estipulou os limites definitivos com duas partes continentais (Serra da Tiririca e Morro das Andorinhas) e uma marinha (Enseada do Bananal), numa área de 2.077 ha. Em 1992, foi considerado pela UNESCO como parte integrante da Reserva da Biosfera da Mata Atlântica. O PEST é formado por um conjunto de montanhas com altitude média aproximada de 250 m: Pedra do Elefante (412 m), Morro do Telégrafo (387 m), Alto Mourão (369 m), Morro do Catumbi (344 m), Morro da Serrinha (277 m), Morro do Cordovil (256 m), Costão de Itacoatiara (217 m), Morro da Penha (128 m) (Barros \& Seoane 1999) e Morro das Andorinhas (196 m), esse último incluído no PEST em 2007. Apresenta rochas do período Pré-Cambriano, com cerca de 600 milhões de anos, compreendendo as unidades geológicas gnaisse facoidal e Cassorotiba (Penha 1999). Os solos são do tipo alissolo crômico, luvissolo hipocrômico, neossolo litólico e formações turfosas (Multiservice 1995), sendo a classificação adaptada de acordo com Zimback (2003). Na classificação de Köppen (Kottek et al. 2006), o clima é do tipo Aw, ou seja, quente e úmido, com estação chuvosa no verão e seca no inverno (maio e junho). A estação chuvosa inicia-se na primavera, culminando no verão entre os meses de dezembro e janeiro, quando ocorre intensa precipitação pluviométrica, correspondendo a $60 \%$ do total anual, porém não excede 171 mm/mês. Em fevereiro, há uma queda no volume das chuvas. Contudo em março, devido à chegada de massas frias, registram-se chuvas intensas. A menor precipitação se dá nos meses de julho e agosto, quando fica abaixo de $60 \mathrm{~mm}$. A temperatura média é de $22^{\circ} \mathrm{C}$, sendo janeiro e fevereiro os meses mais quentes e junho o mais frio (Barbiére \& Coe-Neto 1999). A Serra da Tiririca está inserida no bioma 
Mata Atlântica e sua vegetação é classificada como floresta ombrófila densa ( sensu Veloso et al. 1991), com extensas áreas cobertas pela formação submontana em vários estádios sucessionais. Nos afloramentos rochosos de gnaisse facoidal, observa-se uma vegetação típica de ambientes sujeitos a escassez de água (Barros \& Seoane 1999). A região foi ocupada por antigas fazendas do século XVIII, tendo passado por vários ciclos econômicos que alteraram sua vegetação original, e, atualmente, vem sendo modificada pela ocupação humana visando especulação imobiliária.

\section{Amostragem florística}

Foram realizadas coletas aleatórias de plantas férteis no período de março/1997 a agosto/2007. O material coletado foi herborizado, seco em estufa a $60^{\circ} \mathrm{C}$, e posteriormente incorporado aos Herbários da Faculdade de Formação de Professores da UERJ (RFFP) e do Instituto de Pesquisas Jardim Botânico do Rio de Janeiro (RB). As espécies foram identificadas através de bibliografia especializada, comparações nos herbários fluminenses (RB, GUA, RUSU e HB) e consultas aos especialistas. A lista foi organizada segundo APG II (APG II 2003; Souza \& Lorenzi 2008). A grafia dos nomes das espécies foi confirmada com auxílio de bancos de dados disponíveis na Internet: International Plant Names Index (2008); $\mathrm{W}^{3}$ Tropicos (2008); New York Botanical Garden (2008).

Nesse estudo, foram consideradas trepadeiras as plantas vasculares que utilizam plantas ou outro suporte para ascender, porém são fixas ao solo pelo sistema radicular durante todo seu ciclo de vida (Mueller-Dombois \& Ellenberg 1974; Gentry 1985, 1991). As espécies foram classificadas de acordo com Schnell (1970), com base nos diferentes mecanismos de fixação e ascensão: a) passiva ou escandente (ESC), aquela que apenas se apóia sobre um suporte, sem qualquer mecanismo sensível de aderência; b) volúvel (VOL), aquela que se enrosca de maneira espiralada em torno de um suporte; c) com órgão preensor (PRE), aquela que possui sensibilidade localizada na estrutura responsável pela aderência ao suporte; e d) radicante (RAD), aquela que se apóia ao suporte por meio de raízes adventícias. Também foram classificadas com base na estrutura caulinar, sendo divididas em herbáceas (HERB), quando apresentam caules delgados não lenhosos, e lenhosas (LENH), quando apresentam caules lenhosos.

A análise comparativa entre as áreas com estudos de trepadeiras foi feita através do índice de similaridade de Sørensen pela fórmula $\mathrm{C}_{\mathrm{s}}=2 \mathrm{j} /(\mathrm{a}+\mathrm{b})$, onde $\mathrm{j}=$ número de espécies comuns nas áreas amostradas, $\mathrm{a}=$ número de espécies de cada área a, e b= número de espécies de cada área b (Magurran 1988).

\section{Resultados e Discussão}

Foram registrados 38 famílias, 107 gêneros e 215 espécies com hábito trepador, incluindo 10 espécies ruderais (4,6\%) (Tab. 1). Esses dados correspondem a $20,5 \%$ do total de espécies levantadas para o PEST, estando em segundo lugar na representatividade das formas de crescimento, logo depois do componente arbóreo (Barros 2008). Numa estimativa da representatividade de gêneros e famílias de plantas da floresta ombrófila densa na flora do Rio de Janeiro baseada na coleção do herbário RB (Vaz 1992), as trepadeiras correspondem a 53 famílias e 169 gêneros (22,4\% do total). Estão na segunda posição em termos de número de gêneros, perdendo apenas para as famílias arbóreas/arbustivas. Comparando os dados deste estudo com o trabalho de Vaz (1992), verifica-se que 73,6\% das famílias e $61,5 \%$ dos gêneros de trepadeiras da floresta ombrófila densa do estado do Rio de Janeiro estão representados no PEST.

Leguminosae (com 29 espécies), Sapindaceae (23), Bignoniaceae (22), Malpighiaceae (19) e Apocynaceae (15) foram as famílias com maior número de espécies (Tab. 2, Fig. 1), perfazendo $50,5 \%$ do total de espécies registradas. Segundo Gentry (1991), embora muitas famílias de fanerógamas neotropicais apresentem espécies de trepadeiras, a grande 
Tabela 1 - Listagem florística de trepadeiras do Parque Estadual da Serra da Tiririca (Niterói e Maricá, Rio de Janeiro). Família ( ${ }^{\circ}$ gêneros/ ${ }^{\circ}$ espécies). ESC = passivas ou escandentes; VOL = volúvel; PRE = com órgãos preensores; HERB = herbácea; LENH= lenhosa.

Table 1 -List of the lianas in Serra da Tiririca S tate Park, Niterói and Maricá, RJ. Family ( $n^{\circ}$ genera $/ \mathrm{n}^{\circ}$ species). ESC = passive or scandent; VOL = twining; PRE = with prehensile organs; HERB = herb; LENH= woody.

\begin{tabular}{|c|c|c|c|c|}
\hline Famílias & Espécies & $\begin{array}{l}\text { Forma de } \\
\text { iscenção }\end{array}$ & Hábito & $\begin{array}{l}\text { Material- } \\
\text { testemunho }\end{array}$ \\
\hline Acanthaceae (2/2) & $\begin{array}{l}\text { Mendoncia velloziana Mart. } \\
\text { Thunbergia alata Bojer }\end{array}$ & $\begin{array}{l}\text { VOL } \\
\text { VOL }\end{array}$ & $\begin{array}{l}\text { HERB } \\
\text { HERB }\end{array}$ & $\begin{array}{l}\text { M. C. F. Santos et al. } 508 \\
\text { A. A. M. Barros et al. } 1077\end{array}$ \\
\hline Alstroemeriaceae (1/1) & Bomarea edulis (Tussac) Herb. & VOL & HERB & L. J. S. Pinto et al. 436 \\
\hline Agavaceae (1/1) & Herreria salsaparilha Mart. & $\mathrm{VOL}+\mathrm{PRE}$ & LENH & L. J. S. Pinto et al. 475 \\
\hline Amaranthaceae (2/3) & $\begin{array}{l}\text { Chamissoa altissima (Jack.) Kunth } \\
\text { Chamissoa macrocarpa Kunth } \\
\text { Pfaffia paniculata (Mart.) Kuntze }\end{array}$ & $\begin{array}{l}\text { VOL } \\
\text { VOL } \\
\text { ESC }\end{array}$ & $\begin{array}{l}\text { LENH } \\
\text { LENH } \\
\text { LENH }\end{array}$ & $\begin{array}{l}\text { A. A. M. Barros et al. } 3110 \\
\text { A. A. M. Barros et al. } 3082 \\
\text { A. A. M. Barros et al. } 2216\end{array}$ \\
\hline \multirow[t]{11}{*}{ Apocynaceae (10/15) } & $\begin{array}{l}\text { Condylocarpon isthmicum (Vell.)A. DC. } \\
\text { Ditassa burchellii Hook. \& Arn. } \\
\text { Forsteronia pilosa (Vell.) Müll. Arg. } \\
\text { Forsteronia thyrsoidea (Vell.) Müll. Arg. } \\
\text { Macroditassa grandiflora } \\
\text { (E. Fourn.) Malme }\end{array}$ & $\begin{array}{l}\text { VOL } \\
\text { VOL } \\
\text { VOL } \\
\text { VOL } \\
\text { VOL }\end{array}$ & $\begin{array}{l}\text { LENH } \\
\text { HERB } \\
\text { LENH } \\
\text { LENH } \\
\text { HERB }\end{array}$ & $\begin{array}{l}\text { A. A. M. Barros et al. } 3032 \\
\text { A. A. M. Barros et al. } 1898 \\
\text { A. A. M. Barros et al. } 2945 \\
\text { P. W. Feteira et al. } 212 \\
\text { D. S. D. Araujo et al. } 3151\end{array}$ \\
\hline & $\begin{array}{l}\text { Mandevilla crassinoda } \\
\text { (Gardner) Woodson }\end{array}$ & VOL & HERB & L. O. F. Sousa et al. 123 \\
\hline & $\begin{array}{l}\text { Mandevilla guanabarica Casar. ex } \\
\text { M.F. Salles, Kin-Gouv. \& A.O. Simões }\end{array}$ & VOL & HERB & A. A. M. Barros et al. 679 \\
\hline & Marsdenia loniceroides E. Fourn. & VOL & HERB & L. O. F. Sousa 66 \\
\hline & Marsdenia suberosa (E. Fourn.) Malme & VOL & HERB & A. A. M. Barros et al. 2373 \\
\hline & $\begin{array}{l}\text { Oxypetalum banksii R.Br. ex Schult. } \\
\text { subsp. banksii }\end{array}$ & VOL & HERB & A. A. M. Barros et al. 768 \\
\hline & Peltastes peltatus (Vell.) Woodson & VOL & LENH & A. A. M. Barros et al. 3125 \\
\hline & $\begin{array}{l}\text { Peplonia riedelii (E. Fourn.) } \\
\text { Fontella \& Rapini }\end{array}$ & VOL & HERB & L. J. S. Pinto 433 \\
\hline & Prestonia coalita(Vell.) Woodson & VOL & HERB & A. A. M. Barros et al. 2757 \\
\hline & Prestonia denticulata(Vell.)Woodson & VOL & HERB & A. A. M. Barros et al. 1492 \\
\hline & Prestonia didyma (Vell.) Woodson & VOL & HERB & A. A. M. Barros 856 \\
\hline Arecaceae (1/1) & $\begin{array}{l}\text { Desmoncus polyacanthos Mart. } \\
\text { var. polycanthos }\end{array}$ & $\mathrm{VOL}+\mathrm{PRE}$ & LENH & M. C. F. Santos et al. 154 \\
\hline Aristolochiaceae (1/4) & $\begin{array}{l}\text { Aristolochia cymbifera Mart. \& Zucc. } \\
\text { Aristolochia odora Steud. } \\
\text { Aristolochia raja Mart. } \\
\text { Aristolochia rumicifolia Mart. }\end{array}$ & $\begin{array}{l}\text { VOL } \\
\text { VOL } \\
\text { VOL } \\
\text { VOL }\end{array}$ & $\begin{array}{l}\text { HERB } \\
\text { HERB } \\
\text { HERB } \\
\text { HERB }\end{array}$ & $\begin{array}{l}\text { R. H. P. Andreata et al. } 487 \\
\text { L. J. S. Pinto et al. } 407 \\
\text { A. A. M. Barros et al. } 1562 \\
\text { P. W. Feteira et al. } 221\end{array}$ \\
\hline Asteraceae (2/3) & $\begin{array}{l}\text { Koanophyllon tinctorium Arruda } \\
\text { ex H. Kost. } \\
\text { Mikania hastifolia Baker } \\
\text { Mikania nigricans Gardner }\end{array}$ & $\begin{array}{l}\text { ESC } \\
\text { ESC }\end{array}$ & $\begin{array}{l}\text { HERB } \\
\text { HERB } \\
\text { HERB }\end{array}$ & $\begin{array}{l}\text { R. S. Oliveira et al. } 23 \\
\text { R. S. Oliveira et al. } 53 \\
\text { D. S. D. Araujo } 3763\end{array}$ \\
\hline Bignoniaceae (12/22) & $\begin{array}{l}\text { Adenocalymma bracteatum (Cham.) DC. } \\
\text { Adenocalymma marginatum (Cham.) DC. } \\
\text { Adenocalymma paulistarum } \\
\text { Bureau \& K. Schum. }\end{array}$ & $\begin{array}{l}\text { VOL+PRE } \\
\text { VOL+PRE } \\
\text { VOL+PRE }\end{array}$ & $\begin{array}{l}\text { LENH } \\
\text { LENH } \\
\text { LENH }\end{array}$ & $\begin{array}{l}\text { A. A. M. Barros et al. } 3096 \\
\text { A. A. M. Barros et al. } 2037 \\
\text { R. H. P. Andreata et al. } 354\end{array}$ \\
\hline
\end{tabular}




\begin{tabular}{|c|c|c|c|c|}
\hline Famílias & Espécies & $\begin{array}{l}\text { Forma de } \\
\text { ascenção }\end{array}$ & Hábito & $\begin{array}{l}\text { Material- } \\
\text { testemunho }\end{array}$ \\
\hline & $\begin{array}{l}\text { Adenocalymma trifoliatum (Vell.) } \\
\text { R. C. Laroche }\end{array}$ & $\mathrm{VOL}+\mathrm{PRE}$ & LENH & A. A. M. Barros et al. 2039 \\
\hline & $\begin{array}{l}\text { Anemopaegma chamberlaynii (Sims) } \\
\text { Bureau \& K. Schum. }\end{array}$ & $\mathrm{VOL}+\mathrm{PRE}$ & LENH & K. M. Leal et al. 23 \\
\hline & Anemopaegma prostratum DC. & $\mathrm{VOL}+\mathrm{PRE}$ & LENH & L. J. S. Pinto et al. 399 \\
\hline & Arrabidaea conjugata (Vell.) Mart. & $\mathrm{VOL}+\mathrm{PRE}$ & LENH & A. A. M. Barros et al. 1529 \\
\hline & Arrabidaea leucopogon (Cham.) Sandwith & th VOL+PRE & LENH & A. A. M. Barros 2131 \\
\hline & Arrabidaea rego (Vell.) DC. & $\mathrm{VOL}+\mathrm{PRE}$ & LENH & A. A. M. Barros et al. 767 \\
\hline & Arrabidaea selloi (Spreng.) Sandwith & $\mathrm{VOL}+\mathrm{PRE}$ & LENH & A. A. M. Barros et al. 954 \\
\hline & Arrabidaea subincana Mart. & $\mathrm{VOL}+\mathrm{PRE}$ & LENH & A. A. M. Barros et al. 2336 \\
\hline & Clytostoma binatum (Thunb.) Sandwith & VOL+PRE & LENH & A. A. M. Barros et al. 1736 \\
\hline & $\begin{array}{l}\text { Clytostoma sciuripabulum } \\
\text { Bureau \& K. Schum. }\end{array}$ & $\mathrm{VOL}+\mathrm{PRE}$ & LENH & L. O. F. de Sousa et al. 326 \\
\hline & Glaziovia bauhinioides Bureau exBaill. & PRE & LENH & A. A. M. Barros et al. 2779 \\
\hline & Lundia cordata (Vell.) A. DC. & $\mathrm{VOL}+\mathrm{PRE}$ & LENH & H.P. Moreira et al. 15 \\
\hline & $\begin{array}{l}\text { Mansoa difficilis (Cham.) } \\
\text { Bureau \& K. Schum. }\end{array}$ & $\mathrm{VOL}+\mathrm{PRE}$ & LENH & L. J. S. Pinto et al. 422 \\
\hline & Mansoa lanceolata (DC.) A. H. Gentry & $\mathrm{VOL}+\mathrm{PRE}$ & LENH & N. Coqueiro et al. 270 \\
\hline & $\begin{array}{l}\text { Parabignonia unguiculata (Vell.) } \\
\text { A. H. Gentry }\end{array}$ & $\begin{array}{l}\text { VOL+PRE } \\
+ \text { RAD }\end{array}$ & LENH & A. A. M. Barros et al. 3083 \\
\hline & $\begin{array}{l}\text { Pithecoctenium crucigerum } \\
\text { (L.) A. H. Gentry }\end{array}$ & $\mathrm{VOL}+\mathrm{PRE}$ & LENH & C. Farney $740^{a}$ \\
\hline & Stizophyllum perforatum (Cham.) Miers & s $\mathrm{VOL}+\mathrm{PRE}$ & LENH & R.S. Oliveira et al. 24 \\
\hline & $\begin{array}{l}\text { Tynanthus micranthus Corr. Méllo } \\
\text { ex K. Schum. }\end{array}$ & $\mathrm{VOL}+\mathrm{PRE}$ & LENH & A. A. M. Barros et al. 3084 \\
\hline & $\begin{array}{l}\text { Xylophragma pratense } \\
\text { (Bureau \& K. Schum.) Sprague }\end{array}$ & $\mathrm{VOL}+\mathrm{PRE}$ & LENH & L. O. F. Sousa et al. 207 \\
\hline Boraginaceae (1/1) & Tournefortia gardneri A. DC. & ESC & LENH & A. A. M. Barros et al. 2411 \\
\hline Brassicaceae (1/1) & Capparis lineata Pers. & VOL & HERB & A. A. M. Barros 824 \\
\hline Cactaceae $(1 / 1)$ & Pereskia aculeata Mill. & $\mathrm{ESC}+\mathrm{PRE}$ & LENH & A. A. M. Barros et al. 1480 \\
\hline Celastraceae (1/1) & Hippocratea volubilis L. & VOL+PRE & LENH & A. A. M. Barros et al. 2113 \\
\hline \multirow[t]{2}{*}{ Connaraceae $(1 / 2)$} & Connarus nodosus Baker & $\mathrm{VOL}+\mathrm{PRE}$ & LENH & N. Coqueiro et al. 288 \\
\hline & Connarus rostratus (Vell.) L. B. Smith & VOL & LENH & A. A. M. Barros et al. 2974 \\
\hline \multirow[t]{13}{*}{ Convolulaceae (5/13) } & Argyreia baronii Deroin & VOL & HERB & R. H. P. Andreata et al. 538 \\
\hline & Ipomoea aristolochiifolia G. Don & VOL & HERB & D. S. D. Araujo et al. 3766 \\
\hline & Ipomoea daturiflora Meissn. & VOL & HERB & A. A. M. Barros et al. 2132 \\
\hline & Ipomoea grandifolia (Dammer) O’Donell & $11 \mathrm{VOL}$ & HERB & R. H. P. Andreata 367 \\
\hline & Ipomoea philomega (Vell.) House & VOL & LENH & A. A. M. Barros et al. 2978 \\
\hline & Ipomoea ramosissima (Poir.) Choisy & VOL & HERB & L. O. F. Sousa et al. 279 \\
\hline & Ipomoea tiliacea (Willd.) Choisy & VOL & HERB & A. A. M. Barros 1386 \\
\hline & $\begin{array}{l}\text { Jacquemontia holosericea } \\
\text { (Weinm.) O’Donell }\end{array}$ & VOL & HERB & A. A. M. Barros et al. 3086 \\
\hline & Jacquemontia martii Choisy & VOL & HERB & A. A. M. Barros et al. 689 \\
\hline & Merremia dissecta (Jacq.) Hallier f. & VOL & HERB & L. J. S. Pinto et al. 419 \\
\hline & $\begin{array}{l}\text { Merremia macrocalyx (Ruiz \& Pavon) } \\
\text { O’Donnell }\end{array}$ & VOL & HERB & A. A. M. Barros et al. 698 \\
\hline & Merremia umbellata (L.) Hallier f. & VOL & HERB & A. A. M. Barros et al. 616 \\
\hline & Operculina macrocarpa (L.) Urb. & VOL & HERB & L. O. F. Sousa et al. 314 \\
\hline
\end{tabular}




\begin{tabular}{|c|c|c|c|c|}
\hline Famílias & Espécies & $\begin{array}{l}\text { Forma de } \\
\text { ascenção }\end{array}$ & Hábito & $\begin{array}{l}\text { Material- } \\
\text { testemunho }\end{array}$ \\
\hline \multirow[t]{11}{*}{ Cucurbitaceae (5/11) } & Cayaponia fluminensis (Vell.) Cogn. & VOL+PRE & HERB & L. J. S. Pinto et al. 150 \\
\hline & Cayaponia martiana Cogn. & $\mathrm{VOL}+\mathrm{PRE}$ & HERB & A. A. M. Barros 2211 \\
\hline & Cayaponia trifoliolata Cogn. & VOL+PRE & HERB & L. J. S. Pinto et al. 411 \\
\hline & Cayaponia trilobata Cogn. & $\mathrm{VOL}+\mathrm{PRE}$ & HERB & A. A. M. Barros 2210 \\
\hline & Fevillea trilobata L. & $\mathrm{VOL}+\mathrm{PRE}$ & HERB & T. A. Silva et al. 87 \\
\hline & Melothria cucumis Vell. var. cucumis & $\mathrm{VOL}+\mathrm{PRE}$ & HERB & L. J. S. Pinto et al. 230 \\
\hline & $\begin{array}{l}\text { Melothria fluminensis Gardner } \\
\text { var. fluminensis }\end{array}$ & $\mathrm{VOL}+\mathrm{PRE}$ & HERB & L. J. S. Pinto et al. 340 \\
\hline & Momordica charantia L. & $\mathrm{VOL}+\mathrm{PRE}$ & HERB & A. A. M. Barros et al. 611 \\
\hline & Wilbrandia ebracteata Cogn. & $\mathrm{VOL}+\mathrm{PRE}$ & HERB & L. J. S. Pinto et al. 570 \\
\hline & Wilbrandia glaziovii Cogn. & $\mathrm{VOL}+\mathrm{PRE}$ & HERB & A. A. M. Barros et al. 1935 \\
\hline & Wilbrandia verticillata(Vell.) Cogn. & $\mathrm{VOL}+\mathrm{PRE}$ & HERB & L. J. S. Pinto et al. 572 \\
\hline Dilleniaceae (1/1) & Davilla rugosa Poir. & VOL & LENH & A. A. M. Barros et al. 1512 \\
\hline \multirow[t]{11}{*}{ Dioscoreaceae (1/11) } & Dioscorea altissima Lam. & $\mathrm{VOL}+\mathrm{PRE}$ & LENH & L. J. S. Pinto et al. 801 \\
\hline & Dioscorea cinnamomifolia Hook. & VOL & HERB & L. J. S. Pinto et al. 416 \\
\hline & Dioscorea coronata Hauman & VOL & LENH & A. A. M. Barros et al. 2086 \\
\hline & Dioscorea dodecaneura Vell. & VOL & LENH & A. A. M. Barros et al. 2943 \\
\hline & Dioscorea filiformis Griseb. & VOL & HERB & L. J. S. Pinto et al. 416 \\
\hline & Dioscorea glomerulata Hauman & VOL & LENH & R. H. P. Andreata et al. 406 \\
\hline & Dioscorea mollis Mart. & $\mathrm{VOL}+\mathrm{PRE}$ & LENH & A. A. M. Barros et al. 1530 \\
\hline & Dioscorea ovata Vell. & VOL & HERB & L. J. S. Pinto et al. 416 \\
\hline & $\begin{array}{l}\text { Dioscorea piperifolia Humb. } \\
\text { \& Bonpl. ex Willd. }\end{array}$ & VOL & HERB & A. A. M. Barros et al. 2087 \\
\hline & Dioscorea sinuata Vell. & VOL & HERB & A. A. M. Barros et al. 3088 \\
\hline & Dioscorea sp. & VOL & HERB & P. W. Feteira et al. 206 \\
\hline \multirow[t]{8}{*}{ Euphorbiaceae (3/8) } & Dalechampia alata Müll.Arg. & VOL & HERB & A. A. M. Barros et al. 1758 \\
\hline & Dalechampia brasiliensis Lam. & VOL & HERB & L. J. S. Pinto et al. 134 \\
\hline & Dalechampia convolvuloides Lam. & VOL & HERB & L. J. S. Pinto et al.385 \\
\hline & Dalechampia micromeria Baill. & VOL & HERB & L. J. S. Pinto et al.556 \\
\hline & Dalechampia pentaphylla Lam. & VOL & HERB & L. J. S. Pinto et al.384 \\
\hline & Dalechampia triphylla Lam. & VOL & HERB & A. A. M. Barros 936 \\
\hline & $\begin{array}{l}\text { Romanoa tamnoides (A. Juss.) } \\
\text { A. Radcl.-Sm. }\end{array}$ & VOL & HERB & A. A. M. Barros et al. 2437 \\
\hline & Tragia volubilis L. & VOL & HERB & L. J. S. Pinto et al. 380 \\
\hline Icacinaceae (1/1) & Leretia cordata Vell. & $\mathrm{VOL}+\mathrm{PRE}$ & LENH & A. A. M. Barros et al. 3041 \\
\hline \multirow[t]{11}{*}{ Leguminosae (17/29) } & Abrus precatorius L. & VOL & LENH & A. A. M. Barros et al. 671 \\
\hline & Bauhinia microstachya (Raddi) J.F. Macbr. & r. VOL & LENH & A. A. M. Barros et al. 1978 \\
\hline & Bauhinia radiata Vell. & ESC & LENH & A. A. M. Barros et al. 1932 \\
\hline & Camptosema isopetalum (Lam.) Taub. & VOL & HERB & H. P. Moreira et al. 101 \\
\hline & Canavalia parviflora Benth. & VOL & HERB & A. A. M. Barros et al. 786 \\
\hline & $\begin{array}{l}\text { Centrosema sagittatum } \\
\text { (Humb. \& Bonpl. ex Willd.) Brandegee }\end{array}$ & VOL & HERB & A. A. M. Barros et al. 2293 \\
\hline & Centrosema virginianum (L.) Benth. & VOL & HERB & N. Coqueiro et al. 52 \\
\hline & Chaetocalyx scandens (L.) Urb. & VOL & HERB & K. A. Lúcio et al. 97 \\
\hline & Dalbergia frutescens (Vell.) Britton & VOL & LENH & P. T. Santos et al. 61 \\
\hline & Dalbergia lateriflora Benth. & VOL & LENH & A. A. M. Barros et al. 2993 \\
\hline & Dioclea violacea Mart. ex Benth. & VOL & LENH & L. J. S. Pinto et al. 117 \\
\hline
\end{tabular}




\begin{tabular}{|c|c|c|c|c|}
\hline Famílias & Espécies & $\begin{array}{l}\text { Forma de } \\
\text { ascenção }\end{array}$ & Hábito & $\begin{array}{l}\text { Material- } \\
\text { testemunho }\end{array}$ \\
\hline & Galactia striata (Jacq.) Urb. & VOL & HERB & R.H.P. Andreata 366 et al. \\
\hline & Machaerium aculeatum Raddi & $\mathrm{VOL}+\mathrm{PRE}$ & LENH & A. A. M. Barros et al. 3100 etal. \\
\hline & $\begin{array}{l}\text { Machaerium lanceolatum } \\
\text { (Vell.) J. F. Macbr. }\end{array}$ & VOL & LENH & A. A. M. Barros et al. 1928 et al. \\
\hline & Machaerium oblongifolium Vogel & VOL & LENH & L. J. S. Pinto et al. 187 \\
\hline & Machaerium reticulatum Pers. & $\mathrm{VOL}+\mathrm{PRE}$ & LENH & A. A. M. Barros et al. 1099 \\
\hline & Machaerium violaceum Vogel & VOL & LENH & H. C. Lima 2596 \\
\hline & Mimosa velloziana Mart. & $\mathrm{VOL}+\mathrm{PRE}$ & LENH & A. A. M. Barros et al. 1100 \\
\hline & Mucuna urens (L.) Medik. & VOL & LENH & A. A. M. Barros 2204 \\
\hline & $\begin{array}{l}\text { Piptadenia adiantoides } \\
\text { (Spreng.) J. F. Macbr. }\end{array}$ & VOL & LENH & A. A. M. Barros et al. 3087 \\
\hline & $\begin{array}{l}\text { Senegalia lacerans (Benth.) } \\
\text { Seigler \& Ebinger }\end{array}$ & $\mathrm{ESC}+\mathrm{PRE}$ & LENH & D. S. D. Araujo et al. 3219 \\
\hline & $\begin{array}{l}\text { Senegalia martiusiana (Steud.) } \\
\text { Seigler \& Ebinger }\end{array}$ & $\mathrm{VOL}+\mathrm{PRE}$ & LENH & A. A. M. Barros et al. 3092 et al. \\
\hline & $\begin{array}{l}\text { Senegalia mikanii (Benth.) } \\
\text { Seigler \& Ebinger }\end{array}$ & $\mathrm{VOL}+\mathrm{PRE}$ & LENH & R. H. P. Andreata et al. 616 \\
\hline & $\begin{array}{l}\text { Senegalia pteridifolia (Benth.) } \\
\text { Seigler \& Ebinger }\end{array}$ & $\mathrm{VOL}+\mathrm{PRE}$ & LENH & A. A. M. Barros et al. 2870 \\
\hline & Senegalia sp. 1 & $\mathrm{VOL}+\mathrm{PRE}$ & LENH & N. Coqueiro et al. 26 \\
\hline & Senegalia sp. 2 & VOL+PRE & LENH & N. Coqueiro et al. 39 \\
\hline & Teramnus volubilis Sw. & VOL & HERB & R. S. Oliveira et al. 7 \\
\hline & $\begin{array}{l}\text { Vigna adenantha (G. Mey) } \\
\text { Maréchal, Mascherpa \& Stainer }\end{array}$ & VOL & HERB & A. A. M. Barros et al. 2288 \\
\hline & Vigna speciosa (Kunth) Verdc. & VOL & HERB & A. A. M. Barros 1909 \\
\hline Loganiaceae (1/1) & Strychnos acuta Progel & $\mathrm{VOL}+\mathrm{PRE}$ & LENH & A. A. M. Barros et al. 2368 \\
\hline \multirow[t]{19}{*}{ Malpighiaceae (8/19) } & $\begin{array}{l}\text { Amorimia rigida (A. Juss.) } \\
\text { W. R. Anderson }\end{array}$ & VOL & LENH & A. A. M. Barros et al. 1469 \\
\hline & $\begin{array}{l}\text { Banisteriopsis sellowiana } \\
\text { (A. Juss.) B. Gates }\end{array}$ & VOL & LENH & A. A. M. Barros et al. 2040 \\
\hline & Heteropterys bicolor A. Juss. & VOL & LENH & A. A. M. Barros et al. 2146 \\
\hline & Heteropterys chrysophylla Kunth & VOL & LENH & A. A. M. Barros et al. 928 \\
\hline & $\begin{array}{l}\text { Heteropterys fluminensis } \\
\text { (Griseb.) W. R. Anderson }\end{array}$ & VOL & LENH & P. W. Feteira et al. 5 \\
\hline & Heteropterys leschenaultiana A. Juss. & VOL & LENH & A. A. M. Barros et al. 2797 \\
\hline & Heteropterys pauciflora A. Juss. & VOL & LENH & W. B. Carvalho et al. 204 \\
\hline & Heteropterys sericea (Cav.) A. Juss. & VOL & LENH & A. A. M. Barros et al. 2503 \\
\hline & Heteropterys ternstroemiifolia A. Juss. & VOL & LENH & A. A. M. Barros et al. 2237 \\
\hline & Mascagnia sepium (A. Juss.) Griseb. & VOL & LENH & A. A. M. Barros et al. 1718 \\
\hline & $\begin{array}{l}\text { Niedenzuella acutifolia } \\
\text { (Cav.) W. R. Anderson }\end{array}$ & VOL & LENH & A. A. M. Barros 919 \\
\hline & Peixotoa hispidula A. Juss. & ESC & LENH & T. A. M. Muritiba et al. 66 \\
\hline & $\begin{array}{l}\text { Stigmaphyllon auriculatum } \\
\text { (Cav.) A. Juss. }\end{array}$ & VOL & LENH & A. A. M. Barros et al. 1499 \\
\hline & Stigmaphyllon gayanum A. Juss. & VOL & LENH & P. W. Feteira et al. 93 \\
\hline & Stigmaphyllon lalandianum A. Juss. & VOL & LENH & L. F. Santos et al. 27 \\
\hline & Stigmaphyllon paralias A. Juss. & VOL & LENH & A. A. M. Barros et al. 1540 \\
\hline & Stigmaphyllon tomentosum A. Juss. & VOL & LENH & A. A. M. Barros 918 \\
\hline & Stigmaphyllon vitifolium A. Juss. & VOL & LENH & A. A. M. Barros et al. 777 \\
\hline & Thryallis brachystachys Lindl. & VOL & LENH & A. A. M. Barros 1392 \\
\hline
\end{tabular}




\begin{tabular}{|c|c|c|c|c|}
\hline Famílias & Espécies & $\begin{array}{l}\text { Forma de } \\
\text { ascenção }\end{array}$ & Hábito & $\begin{array}{l}\text { Material- } \\
\text { testemunho }\end{array}$ \\
\hline Marcgraviaceae (1/1) & $\begin{array}{l}\text { Schwartzia brasiliensis (Choise) } \\
\text { Bedell ex Giraldo-Canãs }\end{array}$ & ESC & LENH & R. S. Oliveira et al. 15 \\
\hline \multirow[t]{6}{*}{ Menispermaceae (6/6) } & Abuta convexa (Vell.) Diels. & VOL & LENH & L. J. S. Pinto et al. 799 \\
\hline & $\begin{array}{l}\text { Chondrodendron platiphyllum } \\
\text { (A. St.-Hil.) Miers }\end{array}$ & VOL & LENH & A. A. M. Barros et al. 780 \\
\hline & Disciphania hernandia (Vell.) Barneby & VOL & HERB & A. A. M. Barros et al. 1709 \\
\hline & $\begin{array}{l}\text { Hyperbaena oblongifolia (Eichler) } \\
\text { Chodat \& Hassl. }\end{array}$ & VOL & LENH & A. A. M. Barros et al. 3007 \\
\hline & Odontocarya vitis Miers & VOL & LENH & A. A. M. Barros et al. 1941 \\
\hline & $\begin{array}{l}\text { Ungulipetalum filipendulum } \\
\text { (Mart.) Moldenke }\end{array}$ & VOL & HERB & H. P. Moreira et al. 49 \\
\hline \multirow[t]{3}{*}{ Nyctaginaceae (3/3) } & Bougainvillea spectabilis Willd. & $\mathrm{VOL}+\mathrm{PRE}$ & LENH & A. A. M. Barros et al. 2236 \\
\hline & $\begin{array}{l}\text { Guapira pernambucensis } \\
\text { (Casar.) Lundell }\end{array}$ & ESC & HERB & W. B. Carvalho et al. 20 \\
\hline & Leucaster caniflorus (Mart.) Choisy & VOL & LENH & A. A. M. Barros et al. 1508 \\
\hline \multirow[t]{9}{*}{ Passifloraceae (1/9) } & Passiflora alata Curtis & $\mathrm{VOL}+\mathrm{PRE}$ & HERB & L. J. S. Pinto et al. 424 \\
\hline & Passiflora capsularis L. & $\mathrm{VOL}+\mathrm{PRE}$ & HERB & L. J. S. Pinto et al. 204 \\
\hline & Passiflora edulis Sims & $\mathrm{VOL}+\mathrm{PRE}$ & HERB & A. A. M. Barros et al. 1713 \\
\hline & Passiflora farneyi Pessoa \& Cervi & $\mathrm{VOL}+\mathrm{PRE}$ & HERB & L. J. S. Pinto 159 \\
\hline & Passiflora mucronata Lam. & $\mathrm{VOL}+\mathrm{PRE}$ & HERB & A. A. M. Barros et al. 1927 \\
\hline & Passiflora organensis Gardner & $\mathrm{VOL}+\mathrm{PRE}$ & HERB & L. J. S. Pinto et al. 580 \\
\hline & Passiflora racemosa Brot. & $\mathrm{VOL}+\mathrm{PRE}$ & HERB & A. A. M. Barros et al. 585 \\
\hline & Passiflora setacea DC. & $\mathrm{VOL}+\mathrm{PRE}$ & HERB & L. J. S. Pinto et al. 88 \\
\hline & Passiflora suberosa L. & $\mathrm{VOL}+\mathrm{PRE}$ & HERB & M. G. Santos et al. 947 \\
\hline Poaceae (1/1) & Lasiacis ligulata Hitchc. \& Chase & ESC & HERB & A. A. M. Barros 289 \\
\hline \multirow[t]{2}{*}{ Polygalaceae (1/2) } & Securidaca lanceolata A. St.-Hil. & ESC & LENH & N. Coqueiro et al. 309 \\
\hline & Securidaca ovalifolia A. St.-Hil. \& Moq. & q. ESC & LENH & A. A. M. Barros et al. 2316 \\
\hline Rhamanaceae (1/1) & Reissekia smilacina (Sm.) Steud. & $\mathrm{VOL}+\mathrm{PRE}$ & LENH & L. J. S. Pinto et al. 586 \\
\hline \multirow[t]{3}{*}{ Rubiaceae (3/3) } & $\begin{array}{l}\text { Emmeorhiza umbellata } \\
\text { (Spreng.) K. Schum. }\end{array}$ & VOL & HERB & A. A. M. Barros et al. 773 \\
\hline & Hamelia patens Jacq. & VOL & HERB & A. A. M. Barros et al. 2428 \\
\hline & $\begin{array}{l}\text { Manettia mitis var. fimbriata } \\
\text { (Cham. \& Schltdl.) K. Schum. }\end{array}$ & VOL & HERB & A. A. M. Barros et al. 2532 \\
\hline \multirow[t]{14}{*}{ Sapindaceae (5/23) } & Cardiospermum corindum L. & $\mathrm{VOL}+\mathrm{PRE}$ & HERB & L. J. S. Pinto et al. 342 \\
\hline & Cardiospermum grandiflorum Sw. & $\mathrm{VOL}+\mathrm{PRE}$ & LENH & A. A. M. Barros et al. 1671 \\
\hline & Paullinia coriacea Casar. & $\mathrm{VOL}+\mathrm{PRE}$ & LENH & A. A. M. Barros et al. 1466 \\
\hline & Paullinia fusiformis Radlk. & $\mathrm{VOL}+\mathrm{PRE}$ & LENH & A. A. M. Barros et al. 3051 \\
\hline & Paullinia meliifolia A. Juss. & $\mathrm{VOL}+\mathrm{PRE}$ & LENH & A. A. M. Barros et al. 2140 \\
\hline & Paullinia micrantha Cambess. & $\mathrm{VOL}+\mathrm{PRE}$ & LENH & A. A. M. Barros et al. 2983 \\
\hline & Paullinia racemosa Wawra & $\mathrm{VOL}+\mathrm{PRE}$ & LENH & A. A. M. Barros et al. 2038 \\
\hline & Paullinia trigonia Vell. & $\mathrm{VOL}+\mathrm{PRE}$ & LENH & A. A. M. Barros et al. 1926 \\
\hline & Serjania caracasana (Jacq.) Willd. & $\mathrm{VOL}+\mathrm{PRE}$ & LENH & A. A. M. Barros 1620 \\
\hline & Serjania clematidifolia Cambess. & $\mathrm{VOL}+\mathrm{PRE}$ & LENH & A. A. M. Barros 2206 \\
\hline & Serjania communis Cambess. & $\mathrm{VOL}+\mathrm{PRE}$ & LENH & A. A. M. Barros et al. 2047 \\
\hline & Serjania corrugata Radlk. & $\mathrm{VOL}+\mathrm{PRE}$ & LENH & A. A. M. Barros et al. 1890 \\
\hline & Serjania cuspidata Cambess. & $\mathrm{VOL}+\mathrm{PRE}$ & LENH & A. A. M. Barros et al. 2049 \\
\hline & Serjania dentata Radlk. & $\mathrm{VOL}+\mathrm{PRE}$ & LENH & W. B. Carvalho et al. 265 \\
\hline
\end{tabular}




\begin{tabular}{|c|c|c|c|c|}
\hline Famílias & Espécies & $\begin{array}{l}\text { Forma de } \\
\text { ascenção }\end{array}$ & Hábito & $\begin{array}{l}\text { Material- } \\
\text { testemunho }\end{array}$ \\
\hline & Serjania elegans Cambess. & $\mathrm{VOL}+\mathrm{PRE}$ & LENH & L. T. Vassal et al. 17 \\
\hline & Serjania orbicularis Radlk. & $\mathrm{VOL}+\mathrm{PRE}$ & LENH & M. C. F. Santos et al. 397 \\
\hline & Serjania tenuis Radlk. & VOL+PRE & LENH & A. A. M. Barros et al. 1561 \\
\hline & Thinouia mucronata Radlk. & $\mathrm{VOL}+\mathrm{PRE}$ & LENH & A. A. M. Barros et al. 2372 \\
\hline & $\begin{array}{l}\text { Thinouia scandens (Cambess.) } \\
\text { Triana \& Planchon }\end{array}$ & VOL+PRE & LENH & L. J. S. Pinto et al. 379 \\
\hline & Urvillea glabra Cambess. & $\mathrm{VOL}+\mathrm{PRE}$ & LENH & A. A. M. Barros et al. 1544 \\
\hline & Urvillea laevis Radlk. & $\mathrm{VOL}+\mathrm{PRE}$ & LENH & R. H. P. Andreata et al. 668 \\
\hline & Urvillea stipitata Radlk. & $\mathrm{VOL}+\mathrm{PRE}$ & LENH & A. A. M. Barros et al. 2046 \\
\hline & Urvillea triphylla (Vell.) Radlk. & $\mathrm{VOL}+\mathrm{PRE}$ & LENH & C. Farney 739 \\
\hline \multirow[t]{3}{*}{ Smilacaceae (1/3) } & $\begin{array}{c}\text { Smilax brasiliensis Spreng. } \\
\text { var. grisebachii A. DC. }\end{array}$ & $\mathrm{VOL}+\mathrm{PRE}$ & LENH & A. A. M. Barros et al. 2507 \\
\hline & Smilax quinquenervia Vell. & $\mathrm{VOL}+\mathrm{PRE}$ & LENH & A. A. M. Barros et al. 943 \\
\hline & Smilax subsessiliflora Duham. & $\mathrm{VOL}+\mathrm{PRE}$ & LENH & R. H. P. Andreata et al. 527 \\
\hline Solanaceae $(1 / 1)$ & Solanum alternatopinnatum Steud. & $\mathrm{VOL}+\mathrm{PRE}$ & LENH & K. A. Lúcio et al. 84 \\
\hline \multirow[t]{3}{*}{ Trigoniaceae (1/3) } & $\begin{array}{l}\text { Trigonia eriosperma (Lam.) } \\
\text { Fromm \& E. Santos }\end{array}$ & VOL & LENH & A. A. M. Barros et al. 2670 \\
\hline & Trigonia nivea Cambess. & VOL & LENH & W. B. Carvalho et al. 225 \\
\hline & Trigonia villosa Aubl. & VOL & LENH & A. A. M. Barros et al. 2312 \\
\hline Urticaceae (1/1) & $\begin{array}{l}\text { Urera caracasana (Jacq.) } \\
\text { Gaudich. ex Griseb. }\end{array}$ & ESC & LENH & A. A. M. Barros et al. 612 \\
\hline Violaceae (1/1) & $\begin{array}{l}\text { Anchietea pyrifolia (Mart.) G. Don } \\
\text { var. hilariana (Eichler) Marquete \& Dan. }\end{array}$ & VOL & LENH & A. A. M. Barros et al. 1509 \\
\hline \multirow[t]{5}{*}{ Vitaceae (1/5) } & Cissus paullinifolia Vell. & $\mathrm{VOL}+\mathrm{PRE}$ & HERB & A. A. M. Barros et al. 772 \\
\hline & Cissus serroniana (Glaz.) Lombardi & $\mathrm{VOL}+\mathrm{PRE}$ & HERB & W. B. Carvalho et al. 49 \\
\hline & Cissus simsiana Schult. \& Schult. f. & $\mathrm{VOL}+\mathrm{PRE}$ & HERB & N. Coqueiro et al. 28 \\
\hline & Cissus sulcicaulis (Baker) Planch. & VOL+PRE & LENH & M. C. F. Santos et al. 650 \\
\hline & $\begin{array}{l}\text { Cissus verticillata (L.) Nicolson } \\
\text { \& C. E. Javis }\end{array}$ & $\mathrm{VOL}+\mathrm{PRE}$ & HERB & A. A. M. Barros et al. 2568 \\
\hline
\end{tabular}

diversidade delas está concentrada em poucas famílias. Em outros levantamentos florísticos (Lima et al. 1997; Morellato \& Leitão-Filho 1998; Lombardi et al. 1999; Weiser 2001; Hora \& Soares 2002; Rezende \& Ranga 2005), essas famílias também são representativas em relação ao hábito trepador, mudando apenas a ordem de importância entre elas.

No PEST, as famílias com maior número de gêneros são Leguminosae e Bignoniaceae (Tab. 2), da mesma forma que em Macaé de Cima (Lima et al. 1997). Essas famílias, junto com Asteraceae, são as mais representativas em relação às trepadeiras do estado do Rio de Janeiro (Vaz 1992). Os gêneros com maior número de espécies são Dioscorea (11),

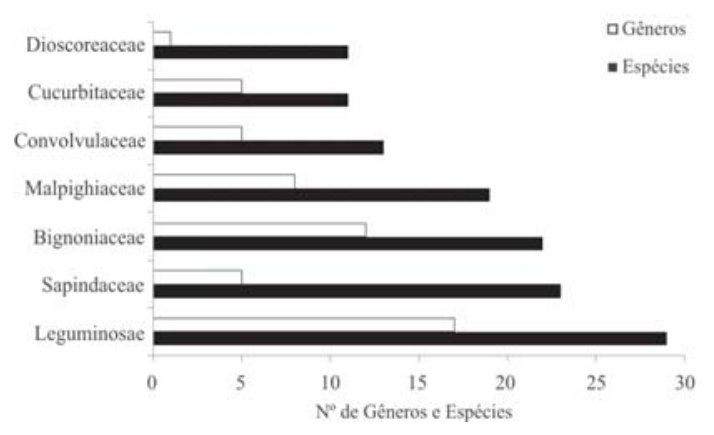

Figura 1 - Número de gêneros e espécies das famílias mais representativas de trepadeiras do Parque Estadual da Serra da Tiririca (Niterói e Maricá, Rio de Janeiro).

Figure 1 - Number of genera and species of the most representative liana families in Serra da Tiririca State Park (Niterói and Maricá, Rio de Janeiro). 
Tabela 2 - Famílias com número de gêneros, espécies, e porcentagem acumulada de espécies no Parque Estadual da Serra da Tiririca (Niterói e Maricá, Rio de Janeiro).

Table 2 - Families with number of genera, species and cumulative percent of species in Serra da Tiririca State Park, Niterói and Maricá, RJ.

\begin{tabular}{llcc}
\hline Famílias & Gêneros & Espécies & $\begin{array}{c}\text { \% } \\
\text { acumulada }\end{array}$ \\
\hline Leguminosae & 17 & 29 & 13,6 \\
Sapindaceae & 5 & 23 & 24,3 \\
Bignoniaceae & 12 & 22 & 34,6 \\
Malpighiaceae & 8 & 19 & 43,5 \\
Apocynaceae & 10 & 15 & 50,5 \\
Convolvulaceae & 5 & 13 & 56,5 \\
Cucurbitaceae & 5 & 11 & 61,7 \\
Dioscoreaceae & 1 & 11 & 66,8 \\
Passifloraceae & 1 & 9 & 71,0 \\
Euphorbiaceae & 3 & 8 & 74,8 \\
Menispermaceae & 6 & 6 & 77,6 \\
Vitaceae & 1 & 5 & 79,9 \\
Aristolochiaceae & 1 & 4 & 81,8 \\
Amaranthaceae & 2 & 3 & 83,2 \\
Asteraceae & 2 & 3 & 84,6 \\
Nyctaginaceae & 3 & 3 & 86,0 \\
Rubiaceae & 3 & 3 & 87,4 \\
Smilacaceae & 1 & 3 & 88,8 \\
Trigoniaceae & 1 & 3 & 90,2 \\
Famílias com & 20 & 21 & 100,0 \\
1 ou 2 espécies & & & \\
\hline
\end{tabular}

Passiflora (9), Serjania (9), Heteropterys (7), Dalechampia, Paullinia, Stigmaphyllon, Senegalia e Ipomoea (6, cada). Esses gêneros representam 30,7\% das espécies amostradas no PEST.

Houve um predomínio de trepadeiras lenhosas $(58,6 \%)$ em relação às herbáceas (41,4\%). Proporção semelhante foi encontrada em florestas estacionais semideciduais de São Paulo (Bernacci \& Leitão-Filho 1996; Morellato \& Leitão-Filho 1998; Udulutsch et al. 2004). Esses dados se contrapõem às estimativas para as florestas tropicais de baixas altitudes, nas quais a representatividade de trepadeiras lenhosas e herbáceas são equivalentes (Gentry 1991).

Seis espécies estão na Lista Brasileira de Espécies da Flora Ameaçada de Extinção (Biodiversitas 2005): Banisteriopsis sellowiana (vulnerável), Heteropterys ternstroemiifolia (criticamente em perigo), Stigmaphyllon vitifolium (vulnerável), Odontocarya vitis (vulnerável), Passiflora farneyi (quase ameaçada) e Ungulipetalum filipendulum (em perigo). Wilbrandia glaziovii é considerada endêmica do estado do Rio de Janeiro, sendo classificada como vulnerável por Klein (1996).

Tabela 3 - Número de espécies de trepadeiras e similaridade florística com outras áreas da Mata Atlântica.

Table 3 -Number of liana species and floristic similarity with other areas of the Atlantic Forest.

\begin{tabular}{|c|c|c|c|c|}
\hline Áreas de Estudo & $\begin{array}{l}\mathrm{N}^{\mathrm{o}} \text { de } \\
\text { espécies }\end{array}$ & $\begin{array}{l}\text { Similaridade } \\
\text { Sørensen (\%) }\end{array}$ & Formação Florestal & Referência \\
\hline Santa Genebra, SP & 136 & 19,5 & $\begin{array}{l}\text { floresta estacional } \\
\text { semidecidual }\end{array}$ & $\begin{array}{l}\text { Morellato \& Leitão- } \\
\text { Filho (1998) }\end{array}$ \\
\hline Rio Claro, Araras, SP & 148 & 17,3 & $\begin{array}{l}\text { floresta estacional } \\
\text { semidecidual }\end{array}$ & Udulutsch et al. (2004) \\
\hline $\begin{array}{l}\text { Estação Ecológica do } \\
\text { Noroeste Paulista, SP }\end{array}$ & 105 & 12,1 & $\begin{array}{l}\text { floresta estacional } \\
\text { semidecidual }\end{array}$ & Rezende \& Ranga (2005) \\
\hline $\begin{array}{l}\text { Parque Estadual de } \\
\text { Vassununga, Gleba Maravilha, SP }\end{array}$ & 120 & 15,0 & $\begin{array}{l}\text { floresta estacional } \\
\text { semidecidual }\end{array}$ & Tibiriçá et al. (2006) \\
\hline Macaé de Cima, Nova Friburgo, RJ & 144 & 16,0 & $\begin{array}{l}\text { floresta ombrófila densa } \\
\text { montana e submontana }\end{array}$ & Lima et al. (1997) \\
\hline Serra da Tiririca, Niterói e Maricá, RJ & J 215 & - & $\begin{array}{l}\text { floresta ombrófila densa } \\
\text { submontana }\end{array}$ & Este estudo \\
\hline
\end{tabular}


O grande número de espécies de trepadeiras no PEST quando comparado a outras áreas de floresta da Região Sudeste (Tab. 3) pode estar associado à fragmentação da vegetação e à concentração de clareiras na região, em ambos os casos em decorrência do grau considerável de perturbação nesse fragmento. Áreas com maior incidência de luz, como clareiras e bordas de mata, favorecem o desenvolvimento de trepadeiras, principalmente herbáceas (Morellato \& Leitão-Filho 1998). A heterogeneidade de habitats, como mencionados por Hora \& Soares (2002), pode estar contribuindo para o grande número de espécies amostradas, já que afloramentos rochosos também foram incluídos neste estudo. Outro fator importante nesse sentido é o esforço de coleta. No PEST, o período para coleta de dados ultrapassa 10 anos, maior do que nos demais estudos.

A grande diversidade de trepadeiras na Serra da Tiririca e a baixa similaridade de sua composição florística com a de outras áreas (Tab. 3) aponta esse tipo de planta como um importante componente estrutural das formações vegetais, não sendo composto apenas por espécies invasoras (veja também Rezende \& Ranga 2005). Situação semelhante pode ser observada tanto em florestas ombrófilas densas como em florestas estacionais semideciduais, demonstrando a necessidade constante de enfocar essas formas de vida em levantamentos florísticos.

\section{Agradecimentos}

Agradecemos aos taxonomistas André Amorim (Malpighiaceae), Elsie F. Guimarães (Trigoniaceae e Boraginaceae), Fábio França (Apocynaceae), Genise Somner (Sapindaceae), Haroldo C. Lima (Leguminosae), João Marcelo A. Braga (Menispermaceae), Jorge Fontella (Apocynaceae - Asclepiadoideae), Luiz José Soares Pinto (Euphorbiaceae), Mário Gomes (Rubiaceae), Michel Barros (Leguminosae Senegalia), Pollyana Wendhausen Feteira (Malpighiaceae), Roberto L. Esteves (Asteraceae) e Robson Dalma Ribeiro (Leguminosae) pela identificação e/ou confirmação do material coletado. Aos revisores anônimos pelas sugestões na redação final do texto.

\section{REFERÊNCIAS BibliográficAS}

APG II. 2003. An update of the Angiosperm Phylogeny Group classification for the orders and families of flowering plants: APG II. Botanical Journal of the Linnean Society 141: 399-436.

Araújo, G. U. C. \& Costa, C. G. 2006. Cambial variant in the stem of Serjania corrugata (Sapindaceae). IAWA Journal 27: 269-280.

Araújo, G. U. C. \& Costa, C. G. 2007. Anatomia do caule de Serjania corrugata Radlk. (Sapindaceae). Acta Botanica Brasilica 21: 489-497.

Barbiére, E. B. \& Coe-Neto, R. 1999. Spatial and temporal variation of rainfall of the east fluminense coast and Atlantic Serra do Mar, State of Rio de Janeiro, Brazil. In: Knoppers, B. A.; Bidone, E. D. \& Abrão, J. J. (eds.). Environmental geochemistry of coastal lagoon systems, Rio de Janeiro, Brazil. Série Geoquímica Ambiental. Vol. 6. Universidade Federal Fluminense, Niterói. Pp. 47-56.

Barros, A. A. M. 2008. Análise florística e estrutural do Parque Estadual da Serra da Tiririca, Niterói E Maricá, RJ, Brasil. Tese de Doutorado. Escola Nacional de Botânica Tropical, Instituto de Pesquisas Jardim Botânico do Rio de Janeiro, Rio de Janeiro. 237p.

Barros, A. A. M. \& Seoane, C. E. S. 1999. A problemática da conservação do Parque Estadual da Serra da Tiririca, Niterói / Maricá, RJ, Brasil. In: Vallejo, L. R. \& Silva, M. T. C. (eds.). Os (des)caminhos do estado do Rio de Janeiro rumo ao século XXI. Anais... Instituto de Geociências da UFF, Niterói. Pp. 114-124.

Bernacci, L. C. \& Leitão-Filho, H. F. 1996. Flora fanerogâmica da floresta da Fazenda São Vicente, Campinas, SP. Revista Brasileira de Botânica 19: 149-164. 
Biodiversitas. 2005. Revisão da lista da flora brasileira ameaçada de extinção. <http:// www.biodiversitas.org.br> Acessado em 02/01/2008.

Brandes, A. F. N. 2007. Anatomia do lenho e dendrocronologia de lianas da família Leguminosae ocorrentes na Mata Atlântica. Dissertação de Mestrado. Escola Nacional de Botânica Tropical, Instituto de Pesquisas Jardim Botânico do Rio de Janeiro, Rio de Janeiro. 94p.

Carlquist, S. 1991. Anatomy of vine and liana stems: a review and synthesis. In: Putz, F. E. \& Mooney, H. A. (eds.). The biology of vines. Cambridge University Press, Cambridge. Pp. 53-72.

Engel, E. E.; Fonseca, R. C. B. \& Oliveira, R. E. 1998. Ecologia de lianas e o manejo de fragmentos florestais. Série Técnica IPEF 12(32): 43-64.

Gentry, A. H. 1985. An ecotaxonomic survey of Panamanian lianas. In: D’Arcy, W. G. \& Correa, A. M. D. (eds.). The botany and natural history of Panama. Missouri Botanical Garden, St. Louis. Pp. 29-42.

Gentry, A.H. 1991. The distribution and evolution of climbing plants. In: Putz, P. E. \& Mooney, H. A. (eds.). The biology of vines. Cambrige University Press, Cambridge. Pp. 3-49.

Gentry, A. H. 1993. A field guide to the families and genera of woody plants of Northwest South America (Colombia, Equador, Peru) with supplementary notes on herbaceous taxa. Conservation International, Washington. 920p.

Hora, R. C. \& Soares, J. J. 2002. Estrutura fitossociológica da comunidade de lianas em uma floresta estacional semidecidual na Fazenda Canchim, São Carlos, SP. Revista Brasileira de Botânica 25: 323-329.

International Plant Names Index. 2008. <http:/ /ipni.org> Acessado em 01/2008.

Klein, V. L. G. 1996. Cucurbitaceae do estado do Rio de Janeiro: Subtribo Melothriinae E.G.O. Muell et F. Pax. Arquivos do Jardim Botânico Rio de Janeiro 34(2): 93-172.
Kottek, M.; Grieser, J.; Beck, C.; Rudolf, B. \& Rubel, F. 2006. World map of the KöppenGeiger climate classification updated. Meteorologische Zeitschrift 15: 259-263.

Leitão-Filho, H. 1995. A vegetação da Reserva de Santa Genebra. In: Morellato, P. \& Leitão-Filho, H. (eds.). Ecologia e preservação de uma floresta tropical urbana, Reserva de Santa Genebra. Editora UNICAMP, Campinas. Pp 19-29.

Lima, H. C.; Lima, M. P. M.; Vaz, A. M. S. F. \& Pessoa, S. V. A. 1997. Trepadeiras da Reserva Ecológica de Macaé de Cima. In: Lima, H. C. \& Guedes-Bruni, R. R. (eds.). Serra de Macaé de Cima: diversidade florística e composição em Mata Atlântica. Jardim Botânico do Rio de Janeiro, Rio de Janeiro. Pp. 75-87.

Lombardi, J. A.; Temponi, L. G. \& Leite, C. A. 1999. Mortality and diameter growth of lianas in semideciduous forest fragment in Southeasterm Brazil. Acta Botanica Brasilica 13: 159-165.

Magurran, A. E. 1988. Ecological diversity and its measurement. Princeton University Press, Princeton. 179p.

Morellato, L. P. C. \& Leitão-Filho, H. F. 1998. Levantamento florístico da comunidade de trepadeiras de uma floresta semidecídua no sudeste do Brasil. Boletim do Museu Nacional do Rio de Janeiro. Série Botânica 103: 1-15.

Mueller-Dombois, D. \& Ellenberg, H. 1974. Aims and methods of vegetation ecology. Ed. John Wiley \& Sons, New York. 547p.

Multiservice. 1995. Avaliação de 10 unidades de conservação ambiental na região metropolitana do Rio de Janeiro. Anexo. 28p.

New York Botanical Garden. 2008. <http:// www.nybg.org> Acessado em 01/2008.

Oliveira, A. N.; Amaral, I. L.; Ramos, M. B. P. \& Formiga, K. M. 2008. Floristic and ecological aspects of large lianas from three forest environments on terra firme in Central Amazonia. Acta Amazonica 38: 421-430. 
Peixoto, A. L. \& Gentry, A. H. 1990. Diversidade e composição florística na mata de tabuleiro na Reserva Florestal de Linhares (Espírito Santo, Brasil). Revista Brasileira de Botânica 13: 19-25.

Peñalosa, J. 1984. Basal branching and vegetative spread in two tropical rain forest lianas. Biotropica 16: 1-9.

Penha, H. M. 1999. A synthesis of geology of the east fluminense coast, state of Rio de Janeiro, Brazil. In: Knoppers, B. A.; Bidone, E. D. \& Abrão, J. J. (eds.). Environmental geochemistry of coastal lagoon systems of Rio de Janeiro, Brazil. Vol. 6. Universidade Federal Fluminense, Niterói. Pp. 3-10.

Pontes, J. A. L. 1987. Serra da Tiririca, RJ. Necessidade de conservação (1 ${ }^{a}$ Contribuição). Boletim da Fundação Brasileira para a Conservação da Natureza 22: 89-94.

Prance, G. T. 1994. A comparison of the efficacy of higher taxa and species numbers in the assessment of biodiversity in the Neotropics. Philosophical Transactions of the Royal Society London, B 345: 89-99.

Putz, F. E. 1984. The natural history of lianas on Barro Colorado Island, Panamá. Ecology 65: 1713-1724.

Rezende, A. A. \& Ranga, N. T. 2005. Lianas da Estação Ecológica do Noroeste Paulista, São José do Rio Preto/Mirassol, SP, Brasil. Acta Botanica Brasilica 19: 273-279.

Ribeiro, J. E. L. S.; Hopkins, M. J. G.; Vicentini, A.; Sothers, C. A.; Costa, M. A. S.; Brito, J. M.; Souza, M. A. D.; Martins, L. H.; Lohmann, L. G.; Assunção, P. A. C. L.; Pereira, E. C.; Silva, C. F.; Mesquita, M. R. \& Procópio, L. C. 1999. Flora da Reserva Ducke. Guia de identificação das plantas vasculares de uma floresta de terra firme na Amazônia Central. INPA, Manaus. 816p.

Sá, C. F. C. 1996. Regeneração em área de floresta de restinga da Reserva Ecológica Estadual de Jacarepiá, Saquarema - RJ. Arquivos do Jardim Botânico do Rio de Janeiro 34: 177-192.
Sá, C. F. C. 2006. Estrutura, diversidade e conservação de angiospermas no Centro de Diversidade de Cabo Frio, estado do Rio de Janeiro. Tese de Doutorado. Universidade Federal do Rio de Janeiro, Rio de Janeiro. 250p.

Schnell, R. 1970. Introdution a la phytogeographie des pays tropicaux. Les flores - Les strutures. Vol. 1. Ed. Gauthier-Villars, Paris. 499p.

Souza, V. C. \& Lorenzi, H. 2008. Botânica sistemática. Guia ilustrado para identificação das famílias de fanerógamas nativas e exóticas no Brasil, baseado em APG II. $2^{\circ}$ edição. Instituto Plantarum, Nova Odessa. 704p.

Stranghetti, V. \& Ranga, N. T. 1998. Levantamento florístico das espécies vasculares de uma floresta estacional mesófila semidecídua da Estação Ecológica de São Paulo de Faria, SP. Revista Brasileira de Botânica 21: 289-298.

Tabanez, A. A. \& Viana, V. M. 2000. Patch structure within Brazilian Atlantic Forest fragments and implications for conservation. Biotropica 32: 925-933.

Tibiriçá, Y. J. A.; Coelho, L. F. M. \& Moura, L. C. 2006. Florística de lianas em um fragmento de floresta estacional semidecidual, Parque Estadual de Vassununga, Santa Rita do Passa Quatro, SP, Brasil. Acta Botanica Brasilica 20: 339-346.

Tropicos.org. 2008. Missouri Botanical Garden. $<$ http://www.tropicos.org > Acessado em 01/2008.

Udulutsch, R. G.; Assis, M. A. \& Picchi, D. G. 2004. Florística de trepadeiras numa floresta estacional semidecídua, Rio Claro Araras, estado de São Paulo, Brasil. Revista Brasileira de Botânica 27: 125-134.

Vaz, A. M. S. F. 1992. Diversidade de plantas vasculares da floresta atlântica do Rio de Janeiro. Boletim do Museu de Biologia Mello Leitão 1: 77-82.

Vaz, A. M. S. F. \& Vieira, C. M. 1994. Identificação de famílias com espécies 
trepadeiras. In: Lima, M. P. M. \& GuedesBruni, R. R. (eds.). Reserva Ecológica de Macaé de Cima, Nova Friburgo, RJ. Aspectos florísticos das espécies vasculares. Vol. 1. Jardim Botânico do Rio de Janeiro, Rio de Janeiro. Pp. 75-82.

Veloso, H. P.; Rangel-Filho, A. L. R. \& Lima, J. C. A. 1991. Classificação da vegetação brasileira adaptada a um sistema universal. IBGE, Rio de Janeiro. 124p.
Weiser, V. L. 2001. Ecologia e sistemática de lianas em um hectare de cerrado stricto sensu da ARIE Cerrado Pé-de-Gigante, Santa Rita do Passa Quatro - SP. Dissertação de Mestrado. Universidade de São Paulo. Ribeirão Preto. 188p.

Zimback, C. R. L. 2003. Classificação de solos. Grupo de Estudos e Pesquisas Agrárias Georreferenciadas, FCA, UNESP, Botucatu. 13p. 